\title{
Scaling similarities of multiple fracturing of solid materials
}

\author{
P. G. Kapiris ${ }^{1}$, G. T. Balasis ${ }^{2}$, J. A. Kopanas ${ }^{1}$, G. N. Antonopoulos ${ }^{1}$, A. S. Peratzakis ${ }^{1}$, and K. A. Eftaxias ${ }^{1}$ \\ ${ }^{1}$ Solid State Section, Physics Department, University of Athens, Greece \\ ${ }^{2}$ GeoForschungsZentrum Potsdam, Germany
}

Received: 13 August 2003 - Revised: 18 December 2003 - Accepted: 19 December 2003 - Published: 25 February 2004

\begin{abstract}
It has recently reported that electromagnetic flashes of low-energy $\gamma$-rays emitted during multi-fracturing on a neutron star, and electromagnetic pulses emitted in the laboratory by a disordered material subjected to an increasing external load, share distinctive statistical properties with earthquakes, such as power-law energy distributions (Cheng et al., 1996; Kossobokov et al., 2000; Rabinovitch et al., 2001; Sornette and Helmstetter, 2002). The neutron starquakes may release strain energies up to $10^{46} \mathrm{erg}$, while, the fractures in laboratory samples release strain energies approximately a fraction of an erg. An earthquake fault region can build up strain energy up to approximately $10^{26} \mathrm{erg}$ for the strongest earthquakes. Clear sequences of kilohertzmegahertz electromagnetic avalanches have been detected from a few days up to a few hours prior to recent destructive earthquakes in Greece. A question that arises effortlessly is if the pre-seismic electromagnetic fluctuations also share the same statistical properties. Our study justifies a positive answer. Our analysis also reveals "symptoms" of a transition to the main rupture common with earthquake sequences and acoustic emission pulses observed during laboratory experiments (Maes et al., 1998).
\end{abstract}

\section{Introduction}

An important question in material science and in geophysics is to identify precursors of macroscopic defects or shocks. We report here some experimental results that may help to gain valuable information in that direction.

Our main tool is the monitoring of the micro-fractures, which occur before the final break-up in the pre-focal area, by recording their kilohertz-megahertz electromagnetic (EM) emissions. We remember, when a heterogeneous material is strained, its evolution toward breaking is characterized by the nucleation and coalescence of micro-cracks before the

Correspondence to: K. A. Eftaxias

(ceftax@phys.uoa.gr) final break-up. Both acoustic as well as EM emissions in a wide frequency spectrum ranging from very low frequencies (VLF) to very high frequencies (VHF), are produced by microcracks, which can be considered as the so-called precursors of general fracture. These precursors are detectable both at a laboratory and a geological scale (Mogi, 1962; Scholz, 1968; Warwick et al., 1982; Gokhberg et al., 1982; Ogawa et al., 1985; Diodati et al., 1991; Fifolt et al., 1993; Anifrani et al., 1995; Petrenko and Gluschenkov, 1996; Garcimartin et al., 1997; Frid et al., 1999; Mavromatou and Hadjicontis, 2001; Bahat et al., 2002). Several experimental results, which illustrate the connection between anomalous VLFVHF EM phenomena and acoustic phenomena with earthquake (EQ) preparation, were presented in a rather comprehensive collection of papers (Hayakawa and Fujinawa, 1994; Hayakawa, 1999; Hayakawa and Molchanov, 2002).

Clear kilohertz-megahertz sequences of EM anomalies have been detected from a few days to a few hours prior to recent destructive EQs in Greece. Recently, we have attempted to establish the hypothesis that the detected EM phenomena are probably precursors emitted during micro-fracturing process from the focal area. This study combines concepts from spectral analysis associated with "intermittent criticality", results from laboratory experiments of rupture, and seismological arguments, as following:

1. Features of these EM precursors are possibly correlated with the corresponding fault model (Eftaxias et al., 2001a; Kapiris et al., 2004) and with the degree of heterogeneity within the pre-focal area (Eftaxias et al., 2000, 2001b, 2004).

2. The evolution of the sequence of the observed radioemissions reveals similarities to that observed in laboratory acoustic and EM emissions during the last stages of failure preparation process in rocks, i.e. about $97 \%-$ $100 \%$ of the fracture strength (Eftaxias et al., 2002). Thus, the idea that the detected kilohertz-megahertz EM emissions came from the focal area is plausible. 
3. A hallmark of EQ preparation process is its extraordinary complexity. Complexity manifests itself in linkages between space and time, generally producing patterns on many scales and the emergence of fractal structures (Newman and Turcotte, 2002). A related consideration is whether the nucleation of EQ is associated with distinctive alterations in these scaling properties, which could be of practical "diagnostic" use. In the frame of this thought a new method to assess the approach of the main shock has been suggested, namely, the monitoring of fractal characteristic of EM emission towards global failure in consecutive time windows. This fractal statistical analysis under the "critical point earthquake model", first proposed in Sornette and Sammis (1995), reveals characteristic signs of approaching the global failure ("critical point") (Kapiris et al., 2002, 2004, 2003; Eftaxias et al., 2003, 2004): (i) emergence of long-range correlations, i.e. the appearance of memory effects, (ii) increase of the spatial correlation in the time series with time; (iii) gradual predominance of large EM events as the main shock is approached, (iv) decrease of the anti-persistence behaviour with time, (v) appearance of persistence properties in the "tail" of the precursors and finally, (vi) the significant divergence of the EM energy release rate with time. These critical features are also compatible with the percolation theory of fracture process (Kapiris et al., 2004).

4. A key parameter of the preparation of fracture is the degree and the nature of disorder. Our results reveal that the interplay between the heterogeneities in the prefocal area and the stress field might be responsible for the observed pattern of the EM time-series. More precisely, the temporal evolution of the Hurst exponent could be understood in the framework of this scenario: "the first anti-persistence part of the precursory EM radiation is triggered by micro-fractures in the highly disordered system that surrounds the, more-or-less, homogeneous backbones within the pre-focal area. The persistence radiation in the "tail" of the emission is thought to be clue to the fracture of the high strength backbones (Kapiris et al., 2004; Eftaxias et al., 2004)".

5. We have uncovered a significant loss of multifractal complexity in the "tail" of the detected EM precursors. We remember that the final part of the precursory EM activity also exhibits persistence behavior. The combination of these two results enhances the scenario that the last stage of the precursory EM activity might be originated in the fracture of the high strength backbones (Kapiris et al., 2004).

It might be argued that beyond any terminology all the above mentioned EM hallmarks distinguish the dynamics in a heterogeneous medium close to its final rupture, and hence, might be considered as candidate precursors of the global instability.

\section{Electromagnetic "critical" pre-fracture hallmarks}

Seismicity is characterized by an extraordinary rich phenomenology and variability, which makes the development of a coherent explanatory and predictive framework very difficult. Most aspects of a new class of models are encompassed by a concept called "intermittent criticality" (Sornette and Sammis, 1995; Saleur et al., 1996a,b; Sammis et al., 1996; Heimpel, 1997; Bowman et al., 1998; Zoller et al., 2001; Zoller and Hainzl, 2002). This approach is an outgrowth of efforts to characterize large earthquakes as a "critical phenomenon", culminating in a large event that is analogous to a kind of "critical point". The fundamental hypothesis of the "critical point earthquake model" is that a large EQ is the end result of a process in which the stress field becomes correlated over increasing long scale-lengths. The largest event possible of the fault network can not occur until regional criticality has been achieved and stress is consequently correlated at all length scales up to size of the region. This large event then destroys criticality on its associated network, creating a period of relative quiescence after which the process repeats by rebuilding correlation lengths towards criticality and the next large event. Thus a large EQ acts as a sort of "critical point" dividing the seismic cycle into a period of growing stress correlations before the great EQ and a relatively uncorrelated stress field after. The critical point hypothesis predicts two different precursory phenomena in space and time: the accelerating moment release and the growth of the spatial correlation length.

The school of "intermittent criticality" implies variations in the seismic activity during a seismic cycle. Consequently, a degree of predictability might then become possible monitoring the fault network. We remember that our main tool is the monitoring of the micro-fractures, which occur before the final break-up in the pre-focal area, by recording their kilohertz-megahertz EM emissions. The mentioned distinctive alterations in the scaling properties of the EM time series as the global instability is approached (see introduction) imply that the evolution of the Earth's crust toward the "critical point" may take place not only in the seismological sense but also in the associated VLF-VHF electromagnetic sense.

Recently, we have studied the precursory EM phenomena in the framework of the "Intermittent Dynamics of Critical Fluctuations" model that reveals the dynamics of critical fluctuations (Contoyiannis and Diakonos, 2000; Contoyiannis et al., 2002). A recent analysis (Contoyiannis et al., 2004) suggests that the EM precursors can be described in terms of intermittent dynamics of type I (Schuster, 1998). The estimated critical exponents classify the phenomenon to the 3D-Ising universality class (Kapiris et al., 2003; Contoyiannis et al., 2004).

The observed behavior of the EM precursors is contrary to what predicted from pure SOC models that imply a system perpetually near global failure (Bak and Tang, 1989; Sornette and Sornette, 1989; Olami et al., 1992, e.g.), hence reducing the degree of predictability of individual earthquakes (Geller et al., 1997). However, these models fail to reproduce 
some important properties of the spatiotemporal clustering of earthquakes observed in real fault systems: they do not show foreshock and aftershock sequences correlated to large earthquakes as well as earthquake swarms (e.g. Hainzl et al., 2000; Main and Al-Kindy, 2002). Grasso and Sornette (1998) have attempted to clarify the notion of SOC and to solve some contradictions between this concept and some observations. Authors try to bridge both the hypothesis of an underlying self-organized critical state and the occurrence of precursory phenomena based on more complex and realistic models or combining self-organized complexity with critical point behaviour (e.g. Huang et al., 1998; Sornette, 2000; Sammis and Smith, 1999; Hainzl et al., 2000; Newman and Turcotte, 2002; Al-Kindy and Main, 2003). These models also imply time-dependant variations in the activity as the main shock is approached, consequently, they may justify the emergence of sort time EM precursors immediately prior to the global instability as well.

More than 100 years since the development of equilibrium statistical mechanics by Boltzmann and Gibbs, the general consensus appears to be that we still do not have an equivalent formalism for predicting the behavior of nonequilibrium systems. It is by now not crystal clear what is and what is not to be called SOC (Turcotte, 2001), and what this concept could add to the understanding of an observed, for example power-law behavior, which constitute a fundamental characteristic of criticality (Maes et al., 1998). Main and Al-Kindy (2002) note that in order to distinguish the difference between competing models of self-organized criticality and intermittent criticality depends on a more precise definition of the two than available at present. Consequently, terms such as "critical point", "criticality", "intermittent criticality" or "self-organized criticality" must be treated with cautiousness due to the fact that these terms are loaded with many different meaning and has been used in distinct ways in several contexts and scientific communities (Sammis and Sornette, 2002; Newman and Turcotte, 2002).

\section{The main directions of the present work}

Keeping in mind the above mentioned cautiousness concerning the terminology we wish to stick to the facts. Here, we attempt to establish more independent arguments in order to evaluate the sequence of the detected EM events as an "EM symptom" of an underlying complex dynamics without characteristic time and length scales. Therefore, turning back to avalanche state of the observed EM phenomena, we concentrate on three types of observations: the amplitude, the quiescent (waiting) time between successive bursts, and the burst lifetime (duration). In particular, we focus on a potential power-law distribution.

Second, a basic reason for our interest in the field of critical phenomena is the striking similarities in behaviour near the "critical point" among systems that are otherwise quite different in nature. Even the numerical values of the "criticalpoint" exponents describing the quantitative nature of the sin- gularities are identical for large groups of apparently physical systems (Stanley, 1999). In this sense, we focus on the question if the pre-seismic EM fluctuations share the same scaling similarities with other emissions observed during multiple fracturing of solid materials.

\section{4 "Gutenberg-Richter type" distribution in pre-fracture "EM events"}

Many forms of scaling invariance appear in seismic phenomena. The most impressive feature is the Gutenberg-Richter law for the magnitude distribution of earthquake:

$\log _{10} N(M>m)=\alpha-b m$,

where $N(M>m)$ is the number of earthquakes with a magnitude greater than $m$ in a specific area and time, while, $\alpha$ and $b$ are two empirical parameters. The constant $\alpha$ is a measure of the intensity of the regional seismicity. The available data indicate that the $b$-value depends on three factors (i) the geometrical properties of the fault, (ii) the physical properties of the medium, and (iii) the stress level of the seismic region. Thus, many of the problems inherent in the identification of precursory seismicity patterns also beset methods designed to detect precursory $b$-value variation . In general, experimental studies indicate that the approach of the main shock is characterized by a jump of the associated $b$-values to lower values (see Sect. 5). It is remarkable that laboratory experiments strongly support this result (Lei et al., 2003).

The Gutenberg-Richter law is at the heart of many models of seismicity. It is thought that such a power-law or fractal distribution may be a fundamental result of multiple fracturing when spontaneously activated micro-cracks tent to coalesce leading, by numerous up-scaling, to a catastrophic failure. Hence, using earthquake terminology (e.g. Maes et al., 1998; Rabinovitch et al., 2001) we focus on a possible EM "Gutenberg-Richter type" distribution in the detected preseismic EM time series.

We concentrate on the kilohertz EM activities observed before the 7 September 1999 Athens earthquake with Ms (Ath) $=5.9$ at Zante station (Eftaxias et al., 2000, 2001a, 2003). These emissions have a rather long duration, i.e. approximately a few days (the data were sampled at $1 \mathrm{~Hz}$ ), thus it provides sufficient data for statistical analysis (Fig. 1a). We note that the pre-seismic accelerating anomalies have been launched through long duration kilohertz EM quiescence, while, they ceased a few hours before the Athens earthquake (Fig. 1b). In this work, we are dealing with the $10 \mathrm{kHz}$ EM time series recording by the magnetic loop antenna having its axis along the $\mathrm{E}-\mathrm{W}$ direction (Fig. 1b).

This "Gutenberg-Richter type" law is expected to be valid for signals with amplitude higher than a threshold connected with some minimal event, i.e. the minimum energy for crack propagation. Here, we limit its validity to signals higher than the experimental noise (Cannelli et al., 1993; Maes et al., 1998). 

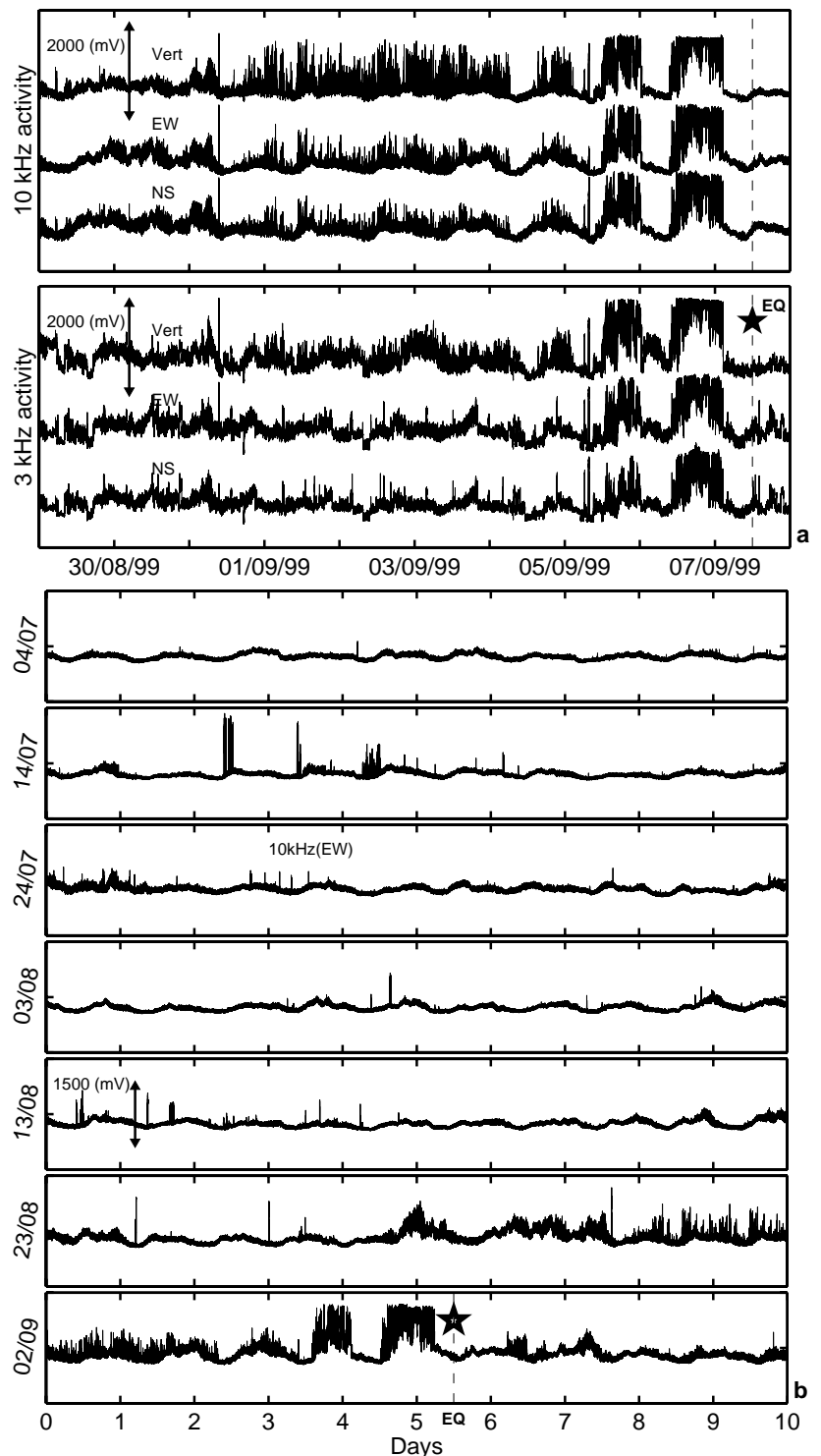

Fig. 1. (a) Electromagnetic anomalies of gradual increasing activity simultaneously recorded at both the $3 \mathrm{kHz}$ and $10 \mathrm{kHz}$ magnetic loop antennas during the last a few days before the $\mathrm{Mw}=5.9$ Athens earthquake on 7 September 1999, 11:56:50 UT. The asterisk indicates the time of the earthquake occurrence. (b) Electromagnetic recordings at the $10 \mathrm{kHz}(\mathrm{E}-\mathrm{W})$ magnetic loop antenna between 4 July 1999 and 11 September 1999. The figure reveals that the accelerating pre-seismic EM activities are embedded in a long duration quiescence period concerning the detection of EM disturbances at the kilohertz frequency band. The signals ceased $\sim 9 \mathrm{~h}$ prior to the main event.

Four ten-days successive intervals of the quiescence period preceding the EM anomaly have been studied and the corresponding amplitude distributions are shown in Fig. 2. Based on these distributions we consider as a background noise level of the times-series the value $A_{\text {noise }} \approx 500 \mathrm{mV}$. Thus, we regard as amplitude $A$ of the precursory "EM events" the difference $A=A_{\text {raw }}-A_{\text {noise }}$.
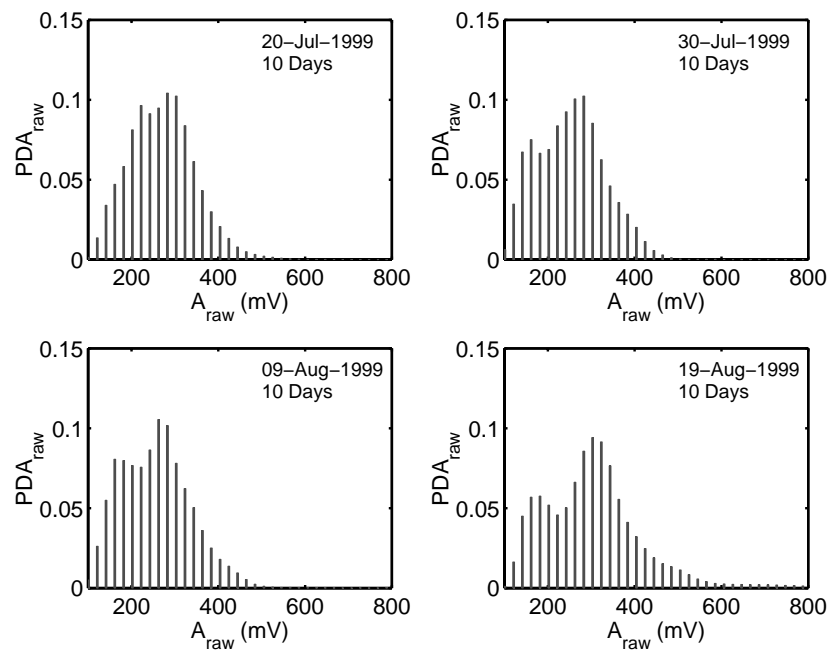

Fig. 2. The probability distribution of amplitude (PDA) of the EM recordings for four ten-day successive intervals of the quiescence period preceding the EM anomaly. These distributions indicate as noise level of the EM times-series the value $A_{\text {noise }} \approx 500 \mathrm{mV}$.

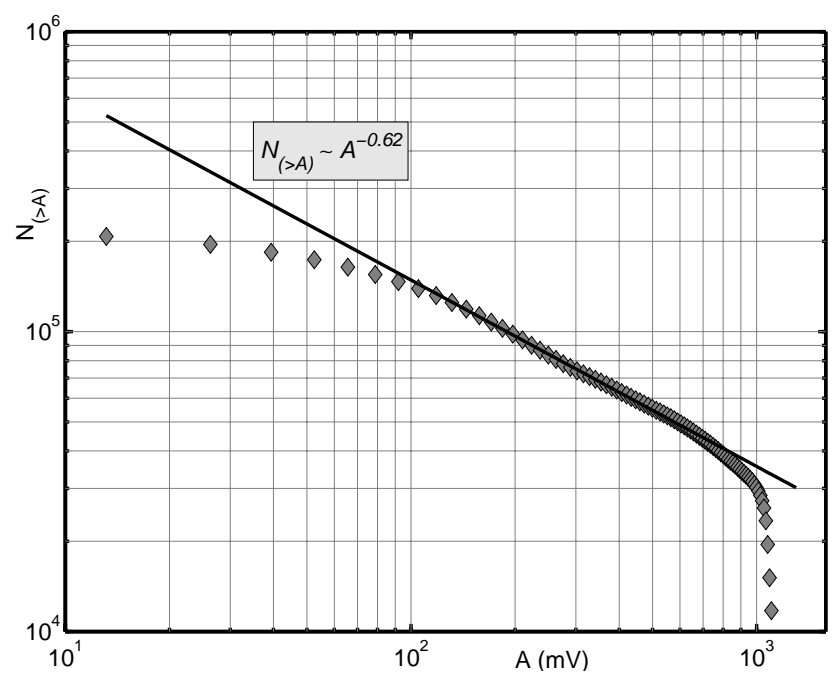

Fig. 3. Number of "EM events" with amplitudes $A=A_{\text {raw }}-A_{\text {noise }}$ (see text) higher than that given by the corresponding abscissa. The continuous line is the least squares fit of the power-law $N(>A)=A^{-b}$ where $b=0.62$. The power-law observed here is the fingerprint of a scale-free activity; an absence of characteristic lengths in the system.

Fig. 3 shows the cumulative number $N(>A)$ of "EM events" (the number of "EM events" having amplitudes larger than $A$ ) vs EM event amplitude $A$. Its main part follows the power-law

$N(>A) \sim A^{-b}$

where $b=0.62$. Hence, the cumulative amplitude distribution of the precursory "EM events" is consistent with a "Gutenberg-Richter-type" law with a $b$-value of 0.62 . The power-law observed here could be interpreted as an EM indication that the system evolves towards the global failure 
without characteristic length scale. This evidence is a typical hallmark of criticality. The following laboratory experimental result strongly enhances this hypothesis.

Rabinovitch et al. (2001) have recently studied the fractal nature of EM radiation induced by uni-axial and tri-axial rock fracture. The analysis of the pre-fracture EM timeseries reveals that the cumulative distribution function of the amplitudes follows a "Gutenberg-Richter type" law with a $b$-value of 0.62 .

We pay attention to close correspondence between laboratory scale EM observations and EM pre-seismic emissions. A power-law-scaling behavior was seen to be due an underlying criticality. The accord of $b$-values in phenomena involving remarkably different scales should be considered as a further hint that the same fracto-electromagnetic critical dynamics hold from the geophysical scale down to the microscopic scale of the sample rheological structure.

Remark: The cumulative number $N(>A)$ of "EM events" vs "EM event" amplitude $A$ is fitted by the power-law $N(>A) \sim A^{-0.62}$ for about one order of magnitude (approximately between $100 \mathrm{mV}$ and $800 \mathrm{mV}$ ). Based exclusively to this information, it is difficult for one to accept the observed power-law as a strong fingerprint of an underlying "scalefree process". However, one should keep in mind the following: (i) The data fit the power-law very well, i.e. the correlation coefficient in the $\log -\log$ representation of the relation $N(>A) \sim A^{-b}$ is $r \sim 1$. (ii) The observed close correspondence between laboratory scale pre-fracture EM events and pre-seismic "EM events". (iii) Accumulated experimental evidence suggests that the EM time series under investigation is in agreement with the critical point earthquake model (Eftaxias et al., 2003; Kapiris et al., 2004; Eftaxias et al., 2004). If we accept these results, then, a power-law relation $N(>A) \sim A^{-b}$ is expected as a pre-fracture signature. (iv) In Sect. 7 we show that the sequence of pre-seismic "EM events" is organized without characteristic time scale. Maslov et al. (1994) have demonstrated that fractal spatial and temporal behaviour are intrinsically related for a broad range of critical phenomena. The authors show that the temporal and spatial activity can be described as different cuts in the same underlying fractal. Thus, if we accept the powerlaw correlations in time, the power-law behaviour in space is also expected. In general, any real data comprise a sample from some unknown population. Based on these considerations it might be concluded that the detected "EM events", due to absorption (or noise) phenomena, are taken from a population of "EM events" which exhibits scaling law behavior in wide temporal scales (see remark Sect. 6). Also, we should keep in mind that the Gutenberg-Richter law for earthquake magnitudes is valid for about seven orders of magnitude.

\section{The observed b-value as a candidate precursory indicator}

It is remarkable that the observed frequency-amplitude relation of pre-seismic "EM events" in comparison agrees with those of Refs. (Kossobokov et al., 2000; Elgazzar, 1998; Gabrielov et al., 2000) for the distribution of earthquake magnitudes in that the $b$-value is of the order of $2 / 3$. More precisely, detailed observations of earthquake catalogs have shown that foreshock sequences and mainshocks are characterized, on average, by a much smaller exponent $b=0.6$, compared to aftershocks, $b=1$ (Meredith, 1990; Knopoff, 2000; Hainzl et al., 2003). Hence, this kind of variation in $b$ is used as a precursory phenomenon (Sobolev et al., 1991). The physical background of this consideration is that the high $b$-values indicate a preponderance of small events, while, the law $b$-values show a preponderance of larger events. Moreover, acoustic emissions in rocks have been studied as a model of natural seismicity. During the deformation of rock in laboratory experiments, small cracking events emerge which radiate elastic waves in a manner similar to earthquake (Scholz, 1968; Ponomarev et al., 1997). These emissions were found to obey the "Gutenberg-Richter type" relation. Acoustic emissions from rock fracturing show an analogous jump of the $b$-value as the main event approaches, i.e. indicate a significant decrease in the level of the observed $b$ values immediately before the critical point: Scholz (1968) has reported that the $b$-values fall between 1 and 0 , with a consistent tendency for $b$ to decrease as stress is raised. Laboratory experiments performed by Ponomarev et al. (1997) also showed a significant fall of the observed $b$-values from 1 to 0.6 just before the global rupture. Lei et al. (2000) have presented the results of an ongoing experimental investigation of compressive failure of homogeneous and heterogeneous rocks. The authors used a rapid data acquisition system to monitor the spatio-temporal distribution of acoustic emission during fault nucleation. They conclude that the $b$ value decreased with increasing acoustic emission rate, from an initial value of about 1.2-1.4; near the final fracturing the $b$-value became as low as about 0.7 .

In summary, from laboratory to geophysical scale the jump of $b$-value to lower values may signalize the approach of the main shock. Based on the aforementioned field and laboratory results it may be said that the appearance of a "Gutenberg-Richter type" distribution of the "EM events" with a $b$-value of 0.62 (that means a preponderance of large "EM events") may indicate that the earthquake process might be in the last stage. We focus on this point.

Wavelet spectral analysis permits quantitative monitoring of the evolution of the transient signals, and hence of the underlying mechanism, by decomposing a time-series to linear super-position of identical predefined mathematical waveforms (wavelets), each with finite duration and narrow frequency content. The global wavelet spectrum from the Morlet continuous wavelet transform was used in order to provide an unbiased and consistent estimation of the true power spectrum of the time-series (Torrence and Compo, 1998). The 


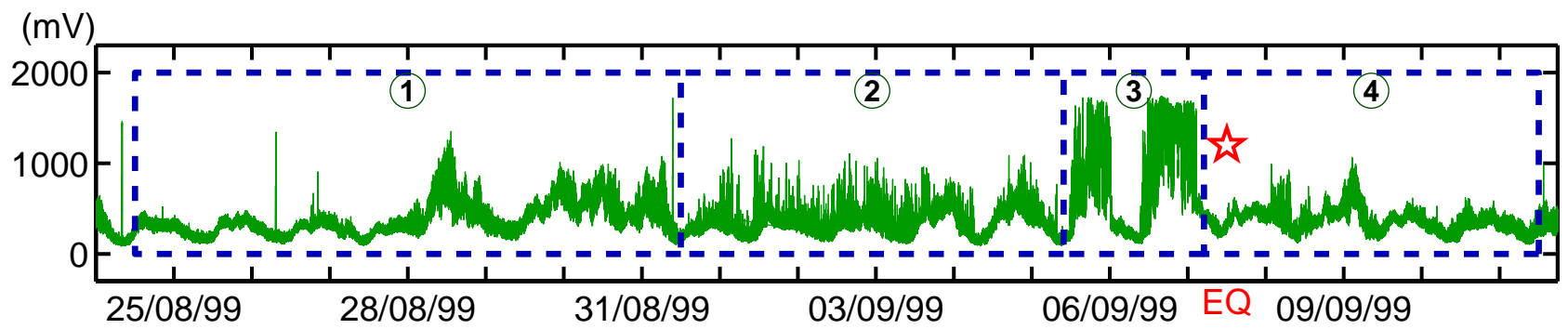

(a)

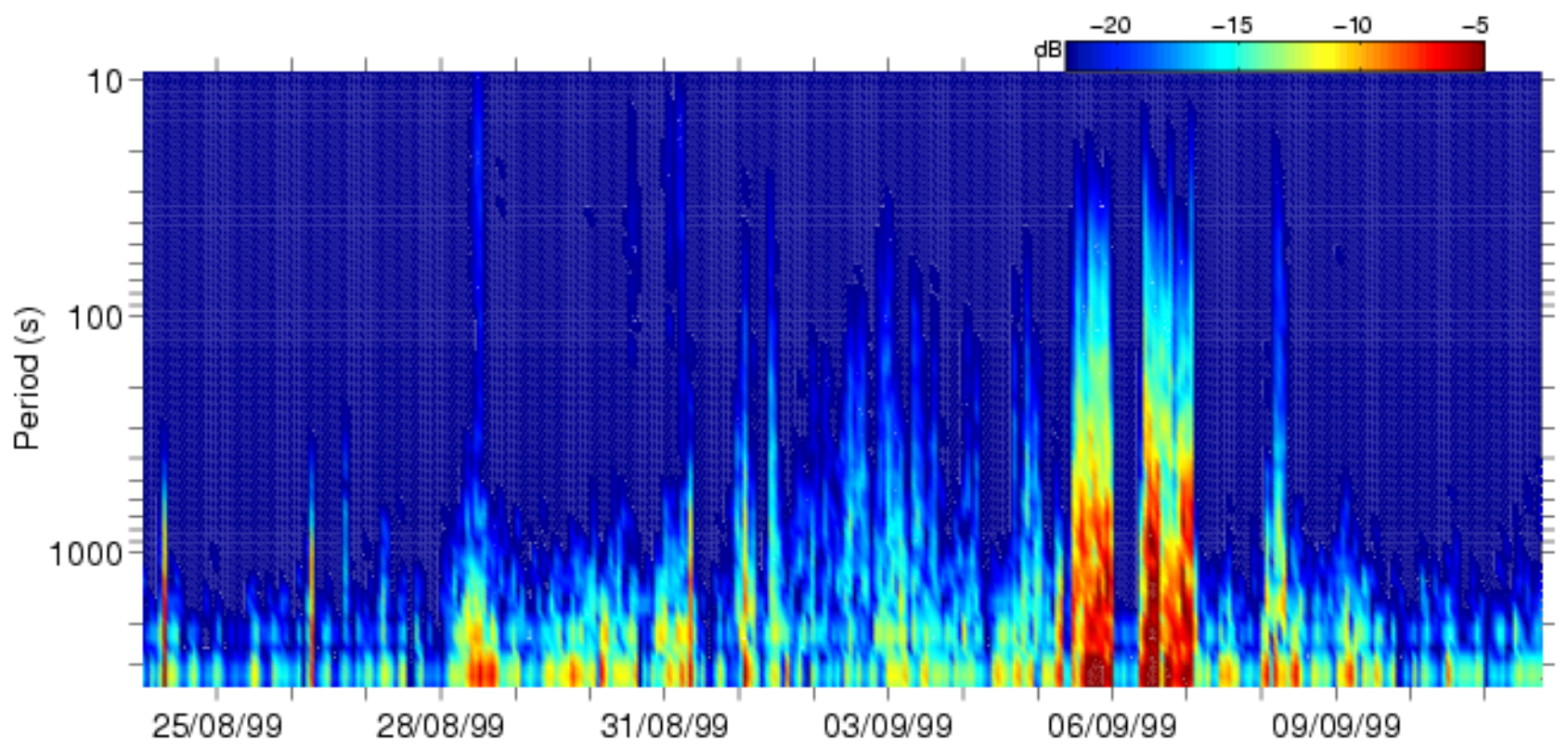

(b)
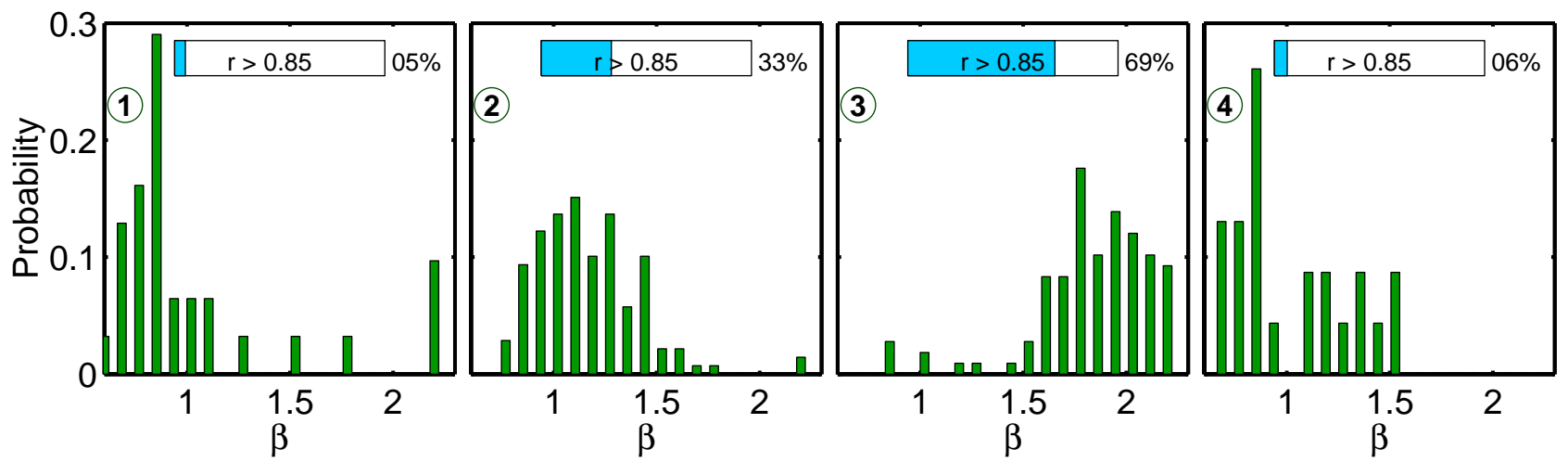

(c)

Fig. 4. (a) The time-series of the $10 \mathrm{kHz}(\mathrm{E}-\mathrm{W})$ magnetic field variations. The star indicates the time of the Athens EQ occurrence. (b) The wavelet power spectrum of the $10 \mathrm{kHz}(\mathrm{E}-\mathrm{W})$ electromagnetic time series between 25 August 1999 and 11 September 1999 . The intensity scale on the top shows colour corresponding to the values of the square spectral amplitudes in arbitrary units. The spectrum reveals both, new higher frequencies are progressively added with time and the amplitude in each frequency (emission rate) increases as the earthquake is approached. (c) Histograms of probability distribution of the exponent $\beta$ calculated on 1024 measurements segments for four consecutive time intervals as marked in (a) taking into account only the segments with good power-law spectrum density $(r>0.85)$. Inserts show the percentage of segments with $r>0.85$. 
wavelet spectrum of the EM signal under investigation is illustrated in Fig. 4b. This figure reveals a progressive shift to higher frequencies. These new components, however, are emerged with small amplitudes. Fig. $4 \mathrm{~b}$ also shows increase of amplitudes in each emission rate as the main shock is approached. It is remarkable that this behaviour mainly characterizes the lower frequencies (emission rates). The question to be answered is whether the system transmits more power at low frequencies than at high frequencies. Further insight on this point can be gathered analyzing the power spectral density.

If the time series of amplitudes of the EM emission is a temporal fractal, then, a power-law spectrum density of the recorded time series is expected (Kapiris et al., 2002): $S(f) \sim f^{-\beta}$. In a $\log S(f)-\log f$ representation the power spectrum is a straight line with linear spectra slope $\beta$. Applying the least square method, we calculate the spectra slope $\beta$ and the linear correlation coefficient $r$ of the fitting associated with successive segments of 1024 samples each and study the distributions of exponent $\beta$ in four consecutive time intervals (Fig. 4a). The power density shows a "colourednoise" type of behaviour, i.e. $\beta>0$. This means that the power spectrum manifests more power at low frequencies than at high frequencies. If all frequencies are equally important (white-noise), we have $\beta=0$. Furthermore, one can recognize that the closer the final stage of seismic process, the larger shift of $\beta$ to higher values (second and third interval in Fig. 4c). The increase in the spectrum slope $\beta$ with time signalizes the gradual prevalence of lower frequency fluctuations in the precursory sequence of "EM events".

By combining the above mentioned information, we conclude that new higher frequencies gradually emerge in the power spectrum; simultaneously, all spectral amplitudes increase as the main shock is approached. The system, however, select to transmit more power at lower frequencies, consequently, their amplitudes increase significantly. Finally, the lower frequencies relatively dominate in the power spectrum. These findings are in agreement with the observed value of $b=0.62$ that indicates the prevalence of large "EM events" in the precursory time series. All these results may reveal the last stage of the earthquake preparation, when due to the high level of clustering of defects, even a new small crack, if it connects large clusters, may generate a large event. The appearance of persistence properties in the tail of the EM precursor enhances the former consideration (Kapiris et al., 2004).

Summarizing the above considerations, we propose that the above mentioned findings demonstrate the basis of similarity between rock deformation experiments in the laboratory and deformation of the crust both in terms of EM waves and elastic waves. We read the observed striking agreement of $b$-values as a further suggestion that the detecting preseismic EM activity is indeed powered by cooperative microfracturing process in the tail of the earthquake generation. We remember that the energy released by observed in the Earth fractures ranges from $10^{26} \mathrm{erg}$, for the strongest earthquakes, down to a fraction of an erg, for laboratory samples.
Thus, the observed similarities may reflect a scenario of an EM critical transition common for a broad class of non-linear systems.

Remark. Figure 3 shows that on both ends of the graph there is a deviation from the Gutenberg-Richter type law. Rabinovitch et al. (2001) have noted a similar slope-change effect for EM pulses obtained during rock fracture in the laboratory. It is remarkable that a number of researchers have noted this slope-change effect for seismic data as values in the smallest and largest ranges deviated from the GutenbergRichter law (Gabrielov et al., 2000). In the range of large amplitudes, the EM $b$-value is significantly higher than in main part (Fig. 3). Scholz (1968); Kossobokov et al. (2000) noted this slope-change effect in the high range of earthquakes and explained it as being due to the maximum energy release and which is limited by the size of the crust and energy density. Concerning our data, we believe such a bend is inevitable since the absorption of the kilohertz signals in the complex structure of the stressed region, and EM energy density limits the maximum EM energy radiated into free space. On the other hand, the observed very low $b$-value for the small amplitude ranges (Fig. 3) might be related to either an incomplete sampling of small events or to noise or to a physical effect governing the process (Rabinovitch et al., 2001).

\section{Characteristics of the Athens earthquake fault pattern}

The principle feature of criticality is the fractal organization in both space and time. Fractals are critical features in the sense that they are not associated to any characteristic scale. From the sub-millimeter scale (Nolen-Hoeksema and Gordon, 1987; Velde et al., 1993; Weiss, 2001) to geophysical scales (Turcotte, 1992; Ouillon et al., 1996) complex fracture patterns have been observed. The earthquakes occur on a fractal structure of faults: fault displacements (Kakimi, 1980), fault and fracture trace lengths (Heffer and Beven, 1990) and fracture apertures (Barton and Zoback, 1992) follow a power-law distribution.

An interesting question that arises concerns the fractal dimension, $D$, of the activated fault. The number of fault ruptures of length larger than $l, N(L>l)$, follows the power-law $N(L>l) \sim l^{-3 b / c}$, where ' $c$ ' a constant (Aki, 1981; Turcotte, 1989). This means that the $b$-value is related to the distribution of rupture fault lengths. For most earthquake studies $c=1.5$ has been appropriate (Kanamori and Anderson, 1975). Consequently, the distribution of rupture fault lengths gives the power-law $N(L>l) \sim l^{-2 b}$.

The previously mentioned relations refer to seismological measurements. The results of the present study suggest that the observed $b$-value in the log-linear frequencyamplitude distribution of electromagnetic events $(b=0.62)$ might be considered representative of the $b$-value of the frequency-magnitude distribution of foreshocks (GutenbergRichter law) associated with the Athens earthquake. Therefore, it might be argued that in the case of Athens earthquake 


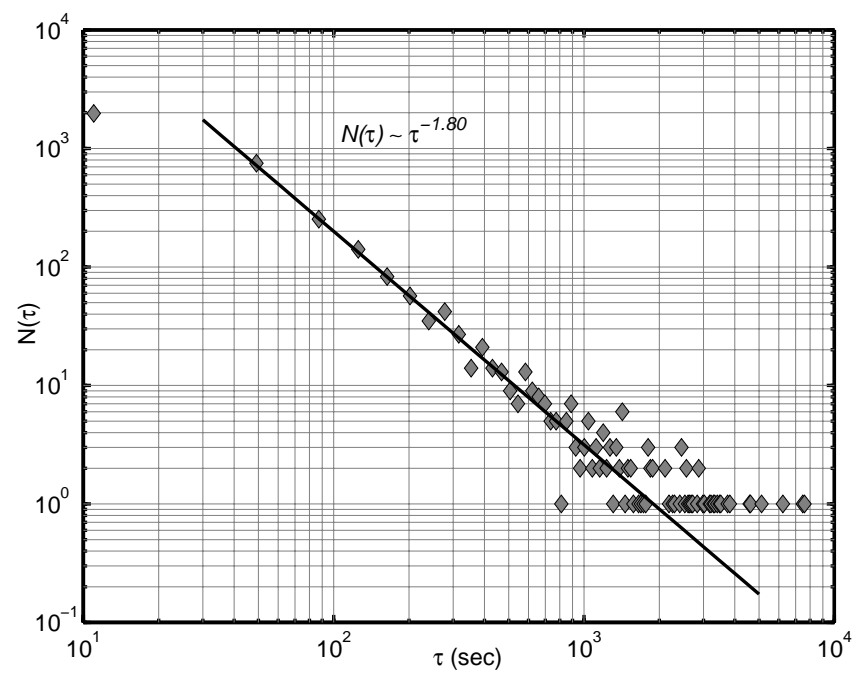

Fig. 5. The distribution of the laminar times $\tau$ between two EM emissions. The strait line is the respective power-law fit. The occurrence of power-law indicates that the system develops critical correlations without characteristic time scales.

the distribution of rupture fault lengths follows the power-law $N(L>l) \sim l^{-1.2}$. In the framework of the standard definition of fractals (Mandelbrot, 1982) one can immediately conclude that the fractal dimension of the fault system associated with the Athens earthquake is $D=1.2$. We note that seismological measurements as well as theoretical studies (e.g. Sahimi, 1993; Sahimi et al., 1993, and references therein) suggest that a surface trace of a single major fault might be characterized by $D \sim 1.2$.

We clarify that the exponent $D$ does not describe the geometrical setting of the rupture faults but it only gives the distribution of rupture fault lengths irrespective of their positions. More information is needed for a full geometrical interpretation of the faults, e.g. the position of the rupture centers (Sornette, 1991).

Remark: An opening crack, due to emitting, diffusing and recombination charge, can act as an EM emitter. Thus, the creation of rupture fault lengths, i.e. fracto-electromagnetic emitters, without characteristic length scale may rationalize the fact that the sequence of pre-seismic EM event is organized in busts that have a distribution of amplitudes that is power-law (see remark in Sect. 4).

\section{Clustering in pre-fracture EM avalanches}

De Boer et al. (1995) argued that a power-law distribution of avalanches is not sufficient enough to claim that a model or a phenomenon is at criticality. They considered that powerlaw correlations in both space and time are at least required. Hence, one can ask how the precursory EM emission correlate in time.

In the following, we investigate the temporal clustering of the pre-seismic EM bursts in terms of burst lifetime (dura-

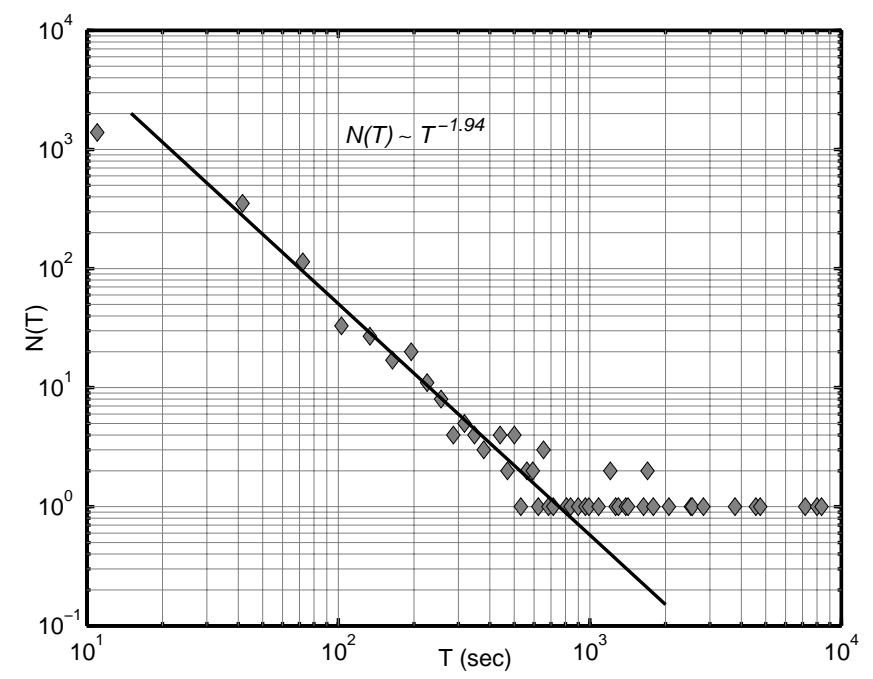

Fig. 6. The distribution of the time duration $T$ of each EM pulse related to the observed pre-fracture EM emission. The strait line is the respective power-law fit. The power-law observed here provides another indication that the systems have developed scaling behavior through a critical process.

tion) and waiting time (time interval between two successive EM events) focusing on a potential power-law distribution.

7.1 Temporal correlations in terms of "waiting-times" statistics

In Fig. 5 the behavior of the distribution $N(\tau)$ of the waiting times $\tau$ is checked. The analysis reveals that, the waiting times display a clear power-law distribution $N(\tau)=A \tau^{-\gamma}$ with $\gamma=1.8$, indicating that the probability of detecting two EM anomalies separated by a time interval $\tau$ decreases as a power-law $\sim \tau^{-1.8}$. Note that Vespignani et al. (1995) measured an exponent $\gamma=1.6$ via acoustic signals from laboratory samples subjected to an external stress.

The occurrence of power-law in the distribution of the waiting times for the precursory EM fluctuations represents a clear indication of the existence of strong correlations between successive EM bursts. Notice that, several authors argue that self organized criticality (SOC) should be discarded as the underlying dynamics if $N(\tau)$, constructed from experimental data, does not decay exponentially (Boffetta et al., 1999). On the contrary, Sanchez et al. (2002) suggest that waiting time statistics cannot be used as a necessary test for SOC behavior in real systems.

\subsection{Temporal correlations in terms of "lifetime" statistics}

The distribution of the lifetimes displayed in the logarithmic plot of Fig. 6. A line with slope -1.9 can fit the experimental results. Thus the distribution of lifetimes follows the scaling law $N(T)=T^{-\delta}$ with $\delta=1.9$. This indicates that the probability $N(T)$ of detecting EM anomalies with duration $T$ decreases on intermediate scales as a power-law $N(T) \sim \tau^{-1.9}$. This finding further supports the hypothesis of the existence 
of strong correlations between EM events in the precursory phenomenon under study.

In summary, the important result to be stressed is that the sequence of pre-seismic EM events is organized in bursts that have a distribution of lifetimes and a distribution of waiting times that are power-laws. We remember that the amplitude and energy distributions of this EM sequence also exhibit power-law behavior. These experimental results constitute a further indication that the system evolves without characteristic time and length scales, which is a typical feature of the existence of an underlying critical dynamics.

\section{Similarities of multiple fracturing from laboratory to geophysical scale}

We remember the proposal that if a great earthquake can be viewed as a critical point, precursors of earthquakes should follow universal power-laws Stanley (1999). Like most critical points, the resulting exponents, although universal, will depend on certain properties in the system: the dimensionality, the range of interactions, and perhaps other aspects.

In Sect. 5, the statistical analysis revealed that the cumulative distribution function of the amplitudes in the pre-seismic EM time series follows the power-law $N(>A) \sim A^{-b}$, where $b=0.62$. The amplitude distribution of the binned data will follow the power-law $N(A) \sim A^{-\zeta}$, where $\zeta=(1+b)$ (Elgazzar, 1998), i.e. $\zeta=1.62$. Remembering that the squared amplitude of the events is proportional to their energy, we can express the distribution function also in terms of the number of events with energy between $E$ and $E+d E$. Will follow the distribution $N(E) \sim E^{-\eta}$, where $\eta=(1+\zeta) / 2$ (Maes et al., 1998), i.e. $\eta=1.31$.

Experiments on, mostly, three-dimensional systems have yielded power-laws for the acoustic energy release; the typical exponent is ranged from 1 to 1.5 (Salminen et al., 2002, and references therein). More precisely, in the following, we compare the energy distribution $N(E) \sim E^{-1.31}$ of the preseismic EM events in binned data with that $N(E) \sim E^{-B}$ obtained from seismicity, acoustic emissions on rock samples, acoustic emissions produced during volcanic activity, and theoretical models reproduced the major dynamical features observed in seismicity. Therefore: (i) The distribution of energies released at any earthquake is well described by the power-law, $N(E) \sim E^{-B}$, where $B \approx 1.4-1.6$. (ii) Diodati et al. (1991) have presented experimental evidence of ultrasonic acoustic emission (AE) from volcanic rocks produced by a survey of the Strobolian activity; the scaling law $N(E) \sim E^{-1.5}$ fits the distribution of the AE bursts. (iii) Petri et al. (1994) have performed the statistical analysis of acoustic emission time series in the ultrasonic frequency range, obtained experimentally from laboratory samples subjected to external uni-axial elastic stress. They found a power-law scaling behavior in the acoustic emission energy distribution $N(E) \sim E^{-B}$ with $B=1.3 \pm 0.1$. We remember that acoustic emission in rocks has been studied as a model of natural seismicity. (iv) Maes et al. (1998) have studied criti- cality in creep experiments on cellular glass. The statistical analysis of the acoustic signals emitted by the sample, while stress is being internally redistributed, shows that the energy distribution follows a power-law, $N(E) \sim E^{-1.5}$. (v) $B=1.5$ is also the value of the exponent measured by Cannelli et al. (1993) via acoustic emission associated to fracture processes in hydrogenated niobium. (vi) Salminen et al. (2002) have reported tensile failure experiments on paper sheets. The acoustic emission energy follows power-law distribution which remains true while the strain rate is varied by more than 2 orders of magnitude. The energy statistics has the exponent $B=1.25$. The authors suggest that a large-scale analogy is earthquakes. (vii) Houle and Sethna (1996) found that the crumpling of paper generates acoustic pulses with a power-law distribution in energy $P(E)=E^{-B}, B=1.3-1.6$. (viii) Chen et al. (1991) have constructed a dynamical model of crack propagation. Taking into account both elastic compression and static friction the authors obtained $B \approx 1.5$. (ix) Gabrielov et al. (2000) have recently developed a "collidingcascades model". This model seemingly exhibits regularities that are common in a wide class of complex hierarchical systems, not necessarily Earth specific. On the basis of this model, the authors suggest that the $B$ value should be 1.53 for main earthquakes. (x) Cowie et al. (1993); Sornette et al. (1994); Cowie et al. (1995) have developed a model of self-organized earthquakes occurring on self-organized faults Their study suggest that the value should be 1.3.

It was found empirically that one could form an analogous of the Mendeleev table if one partitions all critical systems into universality classes (Stanley, 1999). The abovementioned systems with same scaling functions and similar values of critical-point exponents all could belong to the same universality class. This hypothesis strongly reinforces the concept that the detected pre-seismic anomaly corresponds to the emergence of long-range correlations in the stress field preceding the critical point (main rupture). In addition, these findings may indicate that both the pre-seismic mechanical and electromagnetic emissions might be due to the opening cracks as this comes in light from laboratory experiments (Yamada et al., 1989; Fifolt et al., 1993; Petrenko and Gluschenkov, 1996; Mavromatou and Hadjicontis, 2001). In the next section we attempt to further explore the former suggestion.

\section{EM signatures of "critical point" hypothesis in the energy domain}

Observations of the acceleration of seismic moment leading up to large events and "stress shadows" following them have been interpreted as a fundamental evidence that seismic cycles represent the approach and retreat of a fault network from a critical state (Sornette and Sammis, 1995; Sammis and Sornette, 2002). Figure 1a demonstrates a clear accelerating EM energy release rate lasting several days and culminated with the largest precursory signal that ceased a few hours before the main event. In this section we test whether 


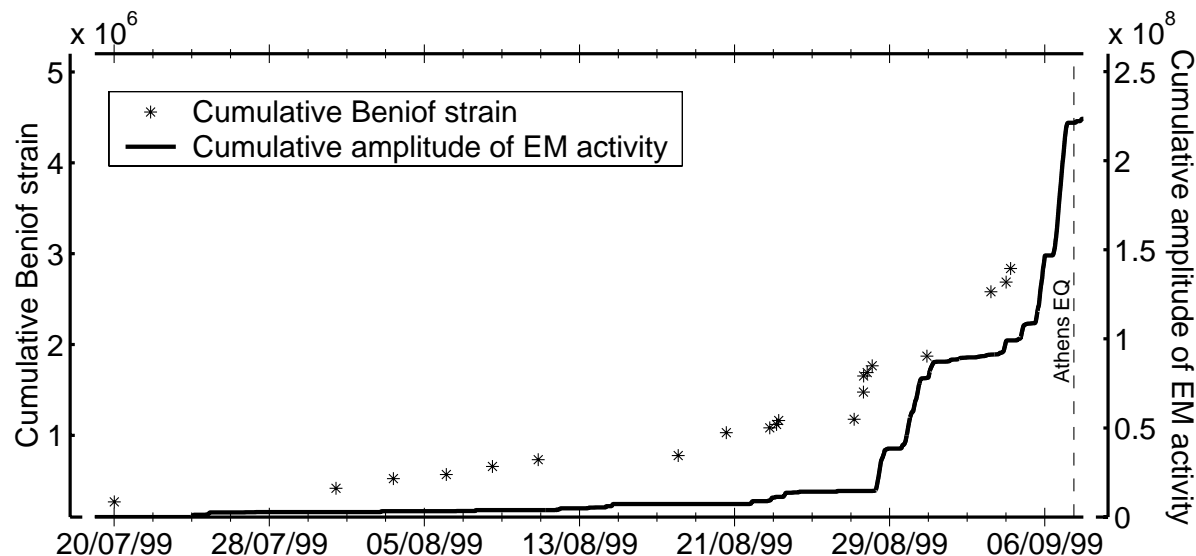

Fig. 7. The Benioff cumulative strain release computed over circle $(R=110 \mathrm{~km})$ on the epicenter of the Athens earthquake as a function of time along with the Benioff cumulative electromagnetic energy release in arbitrary units as a function of time. The strong similarity of the temporal evolution of both the seismic and the EM energy release approaching the main event is pronounced. Provenance of seismicity data is http://www.gein.noa.gr/services/cat.html for the period 10 May 1999-7 September 1999. the temporal evolution of the precursory seismicity associated with the Athens seismic event is correlated with the detected precursory EM activity.

A number of publications have suggested that seismicity over a wide region appears to be modified before a major event. The time-to-failure model is a technique in which a failure function is fitted to a time series of accumulated Benioff strain $\varepsilon(t)=\left(\sum \sqrt{E}\right)(t)$, where $E$ is the energy release of an earthquake, before a large earthquake. In the studies by (Bufe and Varnes, 1993; Sornette and Sammis, 1995; Johansen et al., 1996) premonitory escalation of seismicity is represented by the function

$\varepsilon(t)=\mathcal{A}-\mathcal{B}\left(t_{f}-t\right)^{m}$

where $t_{f}$ is the failure time, while the constants $\mathcal{A}, \mathcal{B}, m$, are positive.

Recently, Tzanis and Makropoulos (2002) have shown that prior to the Athens earthquake, a power-law $\varepsilon(t)=\mathcal{A}-\mathcal{B}\left(t_{f}-\right.$ $t)^{m}$ acceleration of seismic energy release was observed, which could be modelled to yield fair estimates of the critical time, epicentral area and magnitude of the event. The authors have established a critical radius of $110 \mathrm{~km}$. Figure 7 shows the traditional cumulative Benioff strain release $\varepsilon(t)$ as a function of time computed over circle $(R=110 \mathrm{~km})$ on the epicenter of the Athens earthquake, along with the "cumulative Benioff EM energy release" $\left(\sum \sqrt{E}\right)(t) \sim\left(\sum A\right)(t)$ (in arbitrary units) as a function of time, where $E \sim A^{2}$ is the energy of an EM event and $A$ is the amplitude. One can observe the similarity of the temporal evolution of both the mechanical and the EM energy release as the critical point approaches. Thus, the evolution of the quantity $\left(\sum A\right)(t)$ also enables us to monitor the continuous development of the upscaling fracture process through time.

It is remarkable that Rabinovitch et al. (2001) have also reported an accelerating EM energy as the global failure of laboratory sample approaches. The relation $\left(\sum \sqrt{E}\right)(t)=\mathcal{A}-$ $\mathcal{B}\left(t_{f}-t\right)^{m}$ has successfully tested the pre-fracture EMR data, where $E \sim A^{2}$ is the energy of an EM pulse.

These experimental finding further supports the hypothesis of having precursory elastic / EM emissions proportional to the energy of the bonds broken during the cracks evolution (Caldarelli et al., 1996; Bahat et al., 2002). We focus on the subsequent experimental fact that may support the former consideration. The EM phenomenon recorder before the Athens earthquake ends in two very strong signals (Fig. 1a): the first signal contains approximately $20 \%$ of the total EM energy received and the second the remaining $80 \%$ (Eftaxias et al., 2001a). The fault modeling of the Athens earthquake, based on information obtained by radar interferometry (Kontoes et al., 2000), predicts two faults: the main fault segment is responsible for $80 \%$ of the total energy released, with the secondary fault segment for the remaining $20 \%$. Notice that a recent seismic data analysis carried out by M. Kikuchi, indicates that there was probably a subsequent $(\mathrm{Mw}=5.5)$ earthquake after about $3.5 \mathrm{~s}$ of the main event $(\mathrm{Mw}=5.8)$ (Eftaxias et al., 2001a).

As mentioned, observations of the acceleration of seismic moment leading up to large events and "stress shadows" following them have been interpreted as a fundamental evidence that seismic cycles represent the approach and retreat of a fault network for a critical state (Sammis and Sornette, 2002). Therefore, the observed acceleration of the EM emission leading up to EM large event and "EM shadow" following this could be interpreted as an electromagnetic confirmation of the critical point hypothesis.

\section{Thermal Infrared radiances possibly associated with Athens earthquake}

Several studies have done, suggesting on the basis of satellite and ground-based observations that space-time anomalies in the Thermal Infrared (TIR) radiances fields measured by satellite can be related to seismogenic areas distribution, as well as to their activation before earthquakes. The commonly accepted explanation of this correlation relies on the following, simplified, consideration: Earth degassing activity is expected (i) to be generally more intense alongside seismogenic faults (spatially stable anomalies) and (ii) to increase in the pre-seismic phase as far as the process of micro-cracks extensive formation proceeds under a 
continuously increasing stress field (space-time non-stable anomalies) (Tramutoli et al., 2001). Filizzola et al. (2004) have reported that satellite monitoring of thermal anomalies, possibly associated to the Athens earthquake preparation process, have been emerged simultaneously with the launch of the kilohertz electromagnetic anomalies. No TIR anomalies were detected after the earthquake occurrence. This evidence further encourages the seismogenic origin of the observed EM phenomenon.

\section{A possible electromagnetic trace of the Kaizer effect (or Kaizer effect combined with log-periodic oscilla- tions)}

Figure 8 shows the cumulative Benioff type EM energy release $\left(\sum \sqrt{E}\right)(t)$, where $E \sim A^{2}$ is the energy of an EM pulse, during the last few days before the Athens earthquake (grey line), while, the black line shows the least squares fit of the data by a simple power-law $\left(\sum \sqrt{E}\right)(t)=A-B\left(t_{f}-t\right)^{m}$. One can observe a possible trace of oscillations decorating the main power-law behavior. We comment on these oscillations.

1. Irreversible deformation of rocks is accompanied by the Kaizer effect: if the heterogeneous material is loaded, then unloaded before fracture, and loaded again, only a small number of micro-fractures are detected before attaining the previous load. Micro-fracturing activity increases dramatically as soon as the largest previously experienced stress level are exceeded indicating the beginning of further damage in rocks (Chelidze, 1986; Garcimartin et al., 1997). In a recent review article Lavrov (2003) discusses experimentally established features of the Kaizer effect, including mechanism and theoretical models of the phenomenon.

We suggest that the observed oscillations around the main power-law could be considered as a signature of the non-monotonous tectonic stress variation, i.e. as an EM hallmark of the Kaizer effect in the geophysical scale.

2. In a recent series of papers, there has been growing experimental evidence of log-periodic structures decorating the main power-law behavior in acoustic emissions prior to rupture (Anifrani et al., 1995) and in the seismic precursory activity before large earthquakes, (Sornette and Sammis, 1995; Saleur et al., 1996a,b; Varnes and Bufe, 1996; Gluzman and Sornette, 2001). Using computer simulation Sahimi and Arbadi (1996) have shown that, near the global failure point, the cumulative elastic energy released during fracturing of heterogeneous solids follows a power-law with log-periodic corrections to the leading term. The log-periodic oscillations may result from a partial breakdown of the continuous scale-invariance symmetry into a discretescale-invariance (DSI) symmetry (Sornette, 2000), as occurs for instance in hierarchical lattices. Sahimi and

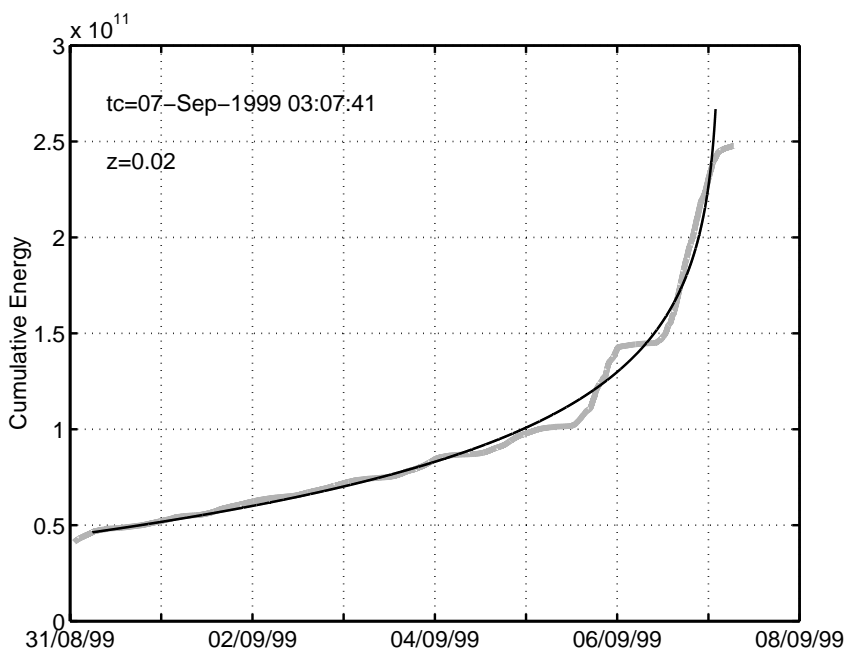

Fig. 8. The gray line shows the temporal evolution of the Benioff type cumulative electromagnetic energy received during the last seven days before the Athens earthquake. The black line fits the data according to the formula $A+B\left(t-t_{c}\right)^{-z}$ where $z=0.02$ and $t_{c}=03: 07: 41$ GMT on 7 September 1999. One can observe that oscillations decorate the main power-law.

Arbadi (1996) suggest that the interplay between the heterogeneities of rock and the stress field generates dynamically such a DSI, and does not have to be present in the rock structure itself. This suggestion is supported by Huang et al. (1997). The authors suggest that short-wavelength Mullins-Sekerka instability, together with strong screening effects, generate spontaneously a DSI in growth phenomena; a signature of this DSI is the presence of log-periodic oscillations correcting the usual power-laws. Log-periodic oscillations have also been documented in the fractal structure of arrays of cracks in geological media (Ouillon et al., 1996).

The Log-periodic oscillations are modulated in frequency with a geometric increase of the frequency on the approach to the time of rupture $t_{c}$. Physically, the oscillations contain information on $t_{c}$. This model gives reliable predictions of the rupture of composite materials and these predictions are now routinely used industrially (Johansen and Sornette, 2000; Sornette and Le Floc'h, 2003).

Figure 8 reveals that the reported precursory oscillations are modulated in frequency with a decrease of frequency on the approach to the time of rupture. Therefore, these oscillations could not be considered as a fingerprint of a logperiodic time-to-failure behaviour. However, it is reasonable that the oscillations around the main power-law could be considered as a signature of the Kaizer effect combined with a log-periodic time-to-failure behavior. 


\section{The limits of similarity in the dynamics of the multiple fracturing}

Neutron stars are believed to have solid crust, which may exhibit tectonic activity analogous to earthquakes. These "starquakes" have been invoked to explain several phenomena associated with neutron stars, such as the flashes of low-energy $\gamma$-ray repeaters (SGRs). A group of 111 starquakes with celestial coordinates 1806-20 were recorded during continuous observation from August 1978 to April 1985. They occurred about 40000 years ago.

Cheng et al. (1996) and Kossobokov et al. (2000) have revealed that the recorded SGR 1806-20 sequence of starquakes shows a power-law energy distribution. We remember that Rabinovitch et al. (2001) have shown that both the Gutenberg-Richter-type and the Benioff strain-release relationship, for earthquakes and starquakes, are shown to extent to the microscale. Finally, we have demonstrated that the pre-seismic EM anomalies are matched with a GutenbergRichter cumulative amplitude distribution with $b=0.62$, a value that (i) measured by Rabinovitch et al. (2001) via electromagnetic emission associated to fracture processes in rocks, and (ii) falls within the observed values for earthquakes.

The above-mentioned sequence of starquakes shows not only the power-law energy distribution, but also "symptoms" of a transition to the main rupture common with earthquake sequences and pre-seismic electromagnetic anomalies. Hence, the association of starquakes into clusters is rather clear from Figs. 1 and 3 in reference (Kossobokov et al., 2000). There, we see a number of clusters before the main event. The former Fig. 3 also shows a power-law increase $\left(\sum \sqrt{E}\right)(t)$ for 111 SGR1806-20 starquakes, while, one can observe a possible trace of logarithmic oscillations. The statistical similarities between earthquakes, SGR events, prefracture EM events from laboratory to geophysical scale may argue for physically similar origins. We remember that the source of a starquake, i.e. the fracture in the neutron star crust, may release strain energy up to $10^{46} \mathrm{erg}$. Thus the limits of similarity in the dynamics of the multiple fracturing are dramatically expanded.

\section{Discussion}

In this study we have performed the statistical analysis of the EM anomalies detected prior to the Athens earthquake. We find that both the amplitudes and energies of the "EM events" follow power-law distributions with exponents consistent with other critical realizations obtained there via real laboratory measurements, theoretical predictions or computer simulations even with earthquakes. We have also monitored the distribution of the time interval between "EM events" and the distribution of the duration of the EM pulses. In both cases we also find a power-law. We propose that the observed power-laws correspond to the emergence of longrange correlations in the pre-focal area. The results also sig- nalize that the system evolves without characteristic time and length scales.

A previous fractal spectral analysis has revealed the following collection of particular features in the precursory kilohertz EM activity: (i) emergence of memory effects, (ii) increase of the spatial correlations in the EM time-series with time, (iii) gradual predominance of large "EM events" as the main shock is approached, (iv) decrease of the antipersistence behavior, (v) appearance of persistence properties in the tail of the precursors, and finally, (vi) significant divergence of the EM energy release rate. These features distinguish the dynamics in a disordered system close to its critical point. The present analysis has added supplementary physically powerful arguments/necessities of an underlying approach to the critical point: (vii) "Gutenberg-Richter type" distribution of "EM events" in terms of amplitude and energy consistent with earthquakes as well as with other critical realizations, (viii) power-law waiting time distribution, (ix) power-law distribution of the durations of the EM pulses, (x) similarity of the temporal evolution of the elastic and the EM energy release.

It might be argued that beyond any terminology all the above mentioned EM fingerprints distinguish the dynamics in a heterogeneous medium close to its final rupture, and hence, might be considered as candidate precursors of the global instability.

There is accumulated evidence that distributed seismicity is a problem in statistical physics: the development and dynamics of fault systems has been studied as a nonequilibrium critical phenomenon. This association has important implications regarding earthquake hazard assessment and forecasting (Kossobokov et al., 2000). In the framework of this consideration, the observed precursory EM peculiarities are consistent with the "Critical Point Earthquake Model" (Sornette and Sammis, 1995).

All the above results yield to the suggestion that the study of precursory EM emissions might be possible to illuminate from another view-point the last stage of the fracture (or earthquake) preparation process. We propose that the appearance of a sequence of EM anomalies that develops in time in agreement with the above mentioned group of physically powerful arguments could give a hint of a considerable probability for a transition to a major rupture.

The conceptual framework of critical phenomena is increasingly finding application in other fields, ranging from chemistry and biology on the one hand to econophysics and liquid water and even internetquakes. Why is this? One possible answer concerns the way in which correlations spread throughout a system comprised of subunits. The critical fracture suggests that as the damage increases, a new "phase" appears, where micro-cracks begin to merge leading to cooperative effects; finally, the main fracture is formed leading to global failure. The challenge is to determine the above mentioned time interval during which short-range correlations evolves to long-range correlations. The collection of critical features/necessities observed in the sequence of EM anomalies detected prior to the Athens earthquake could specify 
such a time interval. This might be indicating that the proposed method of analysis is interesting for readers belonging to different scientific fields.

Acknowledgements. We are grateful to D. Sornette and to the anonymous reviewer for reviewing the original manuscript, whose critical and constructive comments contributed to enhancing the value of the manuscript.

Edited by: J. Büchner

Reviewed by: two referees

\section{References}

Aki, K.: A probabilistic synthesis of precursory phenomena, in: Earthquake Prediction, Maurice Ewing Series 4, edited by Simpson, D. and Richards, P., AGU, Washington, D.C., 566-574, 1981.

Al-Kindy, F. and Main, I.: Testing self-organized criticality in the crust using entropy: A regionalized study of the CMT global earthquake catalog, J. Geophys. Res., 108, 5-1-5-9, 2003.

Anifrani, J.-C., Le Floc'h, C., Sornette, D., and Souillard, B.: Universal log-periodic correction to renormalization group scaling for rupture stress prediction from acoustic emissions, J. Phys. I France, 5, 631-638, 1995.

Bahat, D., Frid, V., Rabinovitch, A., and Palchik, V.: Exploration via electromagnetic radiation and fractographic methods of fracture properties induced by compression in glass-ceramic, International Journal of Fracture, 116, 179-194, 2002.

Bak, P. and Tang, C.: Earthquakes as a self-organized critical phenomenon, J. Geophys. Res., 94, 15 635-15 637, 1989.

Barton, C. and Zoback, M.: Self-similar distribution and properties of macroscopic fractures at depth in crystalline rock in the Cajon Pass scientific borehole, J. Geophys. Res., 97, 5181-5200, 1992.

Boffetta, G., Carbone, V., Giuliani, P., Veltri, P., and Vulpiani, A.: Power laws in solar flares: Self-organized criticality or turbulence?, Phys. Rev. Lett., 83, 4662-4665, 1999.

Bowman, D., Ouillon, G., Sammis, C., Sornette, A., and Sornette, D.: An observational test of the critical earthquake concept, J. Geophys. Res., 103, 24 359-24 372, 1998.

Bufe, C. and Varnes, D.: Predictive modelling of the seismic cycle of the greater San Francisco Bay region, J. Geophys. Res., 98, 9871-9883, 1993.

Caldarelli, G., Di Tolla, F., and Petri, A.: Self-organization and annealed disorder in a fracturing process, Phys. Rev. Lett., 77, 2503-2505, 1996.

Cannelli, G., Cantelli, R., and Cordero, F.: Self-organized criticality of the fracture processes associated with hydrogen precipitation in niobium by acoustic emission, Phys. Rev. Lett., 70, 39233926, 1993.

Chelidze, T.: Percolation theory as a tool for imitation of fracture process in rocks, Pure Appl. Geophys., 124, 731, 1986.

Chen, K., Bak, P., and Obukhov, S.: Self-organized criticality in a crack-propagation model of earthquakes, Physics Review A, 43, 625, 1991.

Cheng, B., Epstein, R., Guyer, R., and Young, C.: Earthquake-like behaviour of soft $\gamma$-ray repeaters, Nature, 382, 518-520, 1996.

Contoyiannis, Y. and Diakonos, F.: Criticality and intermittency in the order parameter space, Phys. Lett. A, 268, 286-292, 2000.
Contoyiannis, Y., Diakonos, F., and Malakis, A.: Intermittent dynamics of critical fluctuations, Phys. Rev. Lett., 89, 35700/135 700/4, 2002.

Contoyiannis, Y., Diakonos, F., Kapiris, P., Peratzakis, A., and Eftaxias, K.: Intermittent dynamics of critical pre-seismic electromagnetic fluctuations, Phys. and Chem. of the Earth, accepted, 2004.

Cowie, A., Sornette, D., and Vanneste, C.: Multifractal scaling properties of a growing fault population, Geophys. J. Int., 122, 457-469, 1995.

Cowie, P., Vanneste, C., and Sornette, D.: Statistical physics model for spatio-temporal evolution of faults, J. Geophys. Res., 98, 21 809-21 821, 1993.

de Boer, J., Jackson, A., and Wettig, T.: Criticality in simple models of evolution, Phys. Rev. E., 51, 1059-1073, 1995.

Diodati, P., Marchesoni, F., and Piazza, S.: Acoustic emission from volcanic rocks: An example of self-organized criticality, Phys. Rev. Let., 67, 2239-2241, 1991.

Eftaxias, K., Kopanas, J., Bogris, N., Kapiris, P., Antonopoulos, G., and Varotsos, P.: Detection of electromagnetic earthquake precursory signals in Greece, Proc. Japan Acad., 76(B), 45-50, 2000.

Eftaxias, K., Kapiris, P., Polygiannakis, J., Bogris, N., Kopanas, J., Antonopoulos, G., Peratzakis, A., and Hadjicontis, V.: Signatures of pending earthquake from electromagnetic anomalies, Geophys. Res. Lett., 28, 3321-3324, 2001a.

Eftaxias, K., Kapiris, P., Polygiannakis, Y., Hadjicontis, V., Chelidze, Z., Zilpimiani, D., and Chelidze, T.: Seismogenic radioemission as a signature of the earthquake preparation process, Journal of the Georgian Geophysical Society, 6, 3-16, $2001 \mathrm{~b}$.

Eftaxias, K., Kapiris, P., Dologlou, E., Kopanas, J., Bogris, N., Antonopoulos, G., Peratzakis, A., and Hadjicontis, V.: EM anomalies before the Kozani earthquake: A study of their behavior through laboratory experiments, Geophys. Res. Lett., 29, 69/1-69/4, 2002.

Eftaxias, K., Kapiris, P., Polygiannakis, J., Peratzakis, A., Kopanas, J., and Antonopoulos, G.: Experience of short term earthquake precursors with VLF-VHF electromagnetic emissions, Natural Hazards and Earth System Sciences, 3, 217-228, 2003.

Eftaxias, K., Frangos, P., Kapiris, P., Polygiannakis, J., Kopanas, J., Peratzakis, A., Skountzos, P., and Jaggard, D.: Review/ model of pre-seismic electromagnetic emissions in terms of fractal electrodynamics, Fractals, in press, 2004.

Elgazzar, A.: An inhomogeneous self-organized critical model for earthquakes, Physica A, 251, 303-308, 1998.

Fifolt, A., Petrenko, F., and Schulson, M.: Preliminary study of electromagnetic emissions from cracks in ice, Phil. Mag. B, 67, 289-299, 1993.

Filizzola, C., Pergola, N., Pietrapertosa, C., and Tramutoli, V.: Robust satellite techniques for seismically active areas monitoring: a sensitivity analysis on September 7th 1999 Athens earthquake, Physics and Chemistry of the Earth, accepted, 2004.

Frid, V., Rabinovitch, A., and Bahat, D.: Electromagnetic radiation associated with induced triaxial fracture in granite, Philos. Mag. Lett., 79, 79-86, 1999.

Gabrielov, A., Keilis-Borok, V., Zaliapin, I., and Newman, W.: Critical transitions in colliding cascades, Phys. Rev. E, 62, 237-249, 2000.

Garcimartin, A., Guarino, A., Bellon, L., and Ciliberto, S.: Statistical analysis of fracture precursors, Phys. Rev. Lett., 79, 32023205, 1997.

Geller, R., Jackson, D., Kagan, Y., and Mulargia, F.: Earthquakes 
cannot be predicted, Science, 275, 1616-1617, 1997.

Gluzman, S. and Sornette, D.: Log-periodic route to fractal functions, Phys. Rev. E, 65, 036 142-1-036 142-19, 2001.

Gokhberg, M., Morgunov, V., and Tomizawa, I.: Experimental measurements of electromagnetic emissions possibly related to earthquake in Japan, J. Geophys. Res., 87(B9), 7824-7828, 1982.

Grasso, J.-R. and Sornette, D.: Testing self-organized criticality by induced seismicity, J. Geophys. Res., 103, 1998.

Hainzl, S., Zoller, G., and Kurths, J.: Seismic quiescence as an indicator for large eartquakes in a system of self-orginized criticality, Geophys. Res. Let., 27, 597-600, 2000.

Hainzl, S., Zoller, G., and Scherbaum, F.: Earthquake clusters resulting from delayed rupture propagation in finite fault segments, J. Geophys. Res. Lett., 108, 2013, 2003.

Hayakawa, M.: Atmospheric and Ionospheric Electromagnetic Phenomena Associated with Earthquakes, Terrapub, Tokyo, 1999.

Hayakawa, M. and Fujinawa, Y.: Electromagnetic Phenomena Related to Earthquake Prediction, Terrapub, Tokyo, 1994.

Hayakawa, M. and Molchanov, O.: Seismo Electromagnetics, Terrapub, Tokyo, 2002.

Heffer, K. and Beven, T.: Scaling relationships in natural fracturesdata theory and applications, Proc. Eur. Petrol. Conf., 2, 367376, 1990.

Heimpel, M.: Critical behavior and the evolution of fault strength during earthquake cycles, Nature, 388, 865-868, 1997.

Houle, P. and Sethna, J.: Acoustic emission from crumpling paper, Phys. Rev. E, 54, 278-283, 1996.

Huang, Y., Ouillon, G., Saleur, H., and Sornette, D.: Spontaneous generation of discrete scale invariance in growth models, Phys. Rev. E, 55, 6433-6447, 1997.

Huang, Y., Saleur, H., Sammis, C., and Sornette, D.: Precursors, aftershocs, criticality and self-organized criticality, Europhys. Lett., 41, 43-48, 1998.

Johansen, A. and Sornette, D.: Critical ruptures, Eur. Phys. J. B, 18, 163-181, http://arXiv.org/abs/cond-mat/0003478, 2000.

Johansen, A., Sornette, D., Wakita, H., Tsunogai, U., Newman, W., and Saleur, H.: Discrete scaling in earthquake precursory phenomena: evidence in the Kobe earthquake, Japan, J. Phys., I France, 6, 1391-1402, 1996.

Kakimi, T.: Magnitude-frequency relation for displacement of minor faults and each significance in crustal deformation, Bull. Geol. Surv. Jap., 31, 467-487, 1980.

Kanamori, H. and Anderson, D.: Theoretical basis of some empirical relations on seismology, Bull. Seism. Soc. Am., 65, 10731095, 1975.

Kapiris, P., Polygiannakis, J., Nomicos, K., and Eftaxias, K.: VHFelectromagnetic evidence of the underlying pre-seismic critical stage, Earth Planets Space, 54, 1237-1246, 2002.

Kapiris, P., Eftaxias, K., Nomikos, K., Polygiannakis, J., Dologlou, E., Balasis, G., Bogris, N., Peratzakis, A., and Hadjicontis, V.: Evolving towards a critical point: A possible electromagnetic way in which the critical regime is reached as the rupture approaches, Nonlin. Proc. Geophys., 10, 511-524, 2003.

Kapiris, P., Eftaxias, K., and Chelidze, T.: The electromagnetic signature of prefracture criticality in heterogeneous media, Phys. Rev. Lett., in press, 2004.

Knopoff, L.: The magnitude distribution of declustered earthquakes in southern California, Proc. Natl. Acad. Sci. USA, 97, 11 880$11884,2000$.

Kontoes, C., Elias, P., Sycioti, O., Briole, P., Remy, D., Sachpazi, M., Veis, G., and Kotsis, I.: Displacement field and fault model for the September 7, Athens earthquake inferred from the ERS2 satellite radar interferometry, Geophys. Res. Lett., 27, 39893992, 2000.

Kossobokov, V., Keillis-Borok, V., and Cheng, B.: Similarities of multiple fracturing on a neutron star and on Earth, Phys. Rev. E, 61, 3529-3533, 2000.

Lavrov, A.: The Kaizer effect in rocks: principle and stress estimation techniques, Int. J. Rock Mech. Mining Sciences, 40, 151$171,2003$.

Lei, X., Nishizawa, O., Kusunose, K., Cho, A., Satoh, T., and Nishizawa, O.: Compressive failure of mudstone samples containing quartz veins using rapid ae monitoring: the role of asperities, Tectonophysics, 328, 329-340, 2000.

Lei, X., Kusunose, K., Satoh, T., and Nishizawa, O.: The hierarchical rupture process of a fault: an experimental study, Phys. Earth Planet. Inter., 137, 213-20-8, 2003.

Maes, C., Moffaert, A., Frederix, H., and Strauven, H.: Criticality in creep experiments on cellular glass, Phys. Rev. B, 57, 49874990, 1998.

Main, I. and Al-Kindy, F.: Entropy, energy and proximity to criticality in global earthquake populations, Geophys. Res. Lett., 29, 25/1-25/4, 2002.

Mandelbrot, B.: The fractal geometry of nature, Freeman, New York, 1982.

Maslov, S., Paczuski, M., and Bak, P.: Avalanches and 1/f noise in evolution and growth models, Phys. Rev. Lett., 73, 2162, 1994.

Mavromatou, C. and Hadjicontis, V.: Laboratory investigation of the electric signals preceding the fracture of crystalline insulators, in: Earthquake thermodynamics and phase transformations in the earth's interior, edited by Teisseyre, R. and Majewski, E., 501-517, Academic Press, 2001.

Meredith, P.: Fracture and failure of brittle polycrystals: an overview, in Deformation Processes in Minerals, Ceramics and Rocks, edited by Barder, D. and Meredith, P. 5-41, Unwin Hyman, London, 1990.

Mogi, K.: Magnitude frequency relation for elastic shocks accompanying fractures of various materials and some related problems in earthquakes, Bull. Earthquake Res. Instit., 40, 831-853, 1962.

Newman, W. and Turcotte, D.: A simple model for the erthquake cycle combining self-organized complexity with critical point behavior, Nonlin. Proc. Geophys., 9, 453-461, 2002.

Nolen-Hoeksema, R. and Gordon, R.: Optical detection of crack patterns in the opening-mode fracture of marble, Int. J. Rock Mech. Min. Sci., 98, 11 935-11 940, 1987.

Ogawa, T., Oike, K., and Miura, T.: Electromagnetic radiations from rocks, J. Geophys. Res., 90, 6245-6249, 1985.

Olami, Z., Feder, H., and Christensen, K.: Self organized criticality in a continuous, nonconservative cellular automaton modeling earthquakes, Phys. Rev. Let., 68, 1244-1247, 1992.

Ouillon, G., Castaing, C., and Sornette, D.: Hierarchical geometry of faulting, J. Geophys. Res., 101, 5477-5487, 1996.

Petrenko, V. and Gluschenkov, O.: Crack velocities in freshwater and saline ice, J. Geophys. Res., 101(B5), 11 541-11 551, 1996.

Petri, A., Paparo, G., Vespignani, A., Alippi, A., and Constantini, M.: Experimental evidence for critical dynamics in microfracturing processes, Phys. Rev. Let, 73, 3423-3426, 1994.

Ponomarev, A., Zavyalov, A., Smirnov, V., and Lockner, D.: Physical modelling of the formation and evolution of seismically active fault zones, Tectonophysics, 277, 57-81, 1997.

Rabinovitch, A., Frid, V., and Bahat, D.: Gutenberg-Richter-type relation for laboratory fracture-induced electromagnetic radiation, Phys. Rev. E, 65, 11 401/1-11 401/4, 2001.

Sahimi, M.: Flow phenomena in rocks: from continuum models to 
fractals, percolation, cellular automata, and simulated annealing, Rev. Mod. Phys., 65, 1393-1534, 1993.

Sahimi, M. and Arbadi, S.: Scaling laws for fracture of heterogeneous materials and rock, Phys. Rev. Lett., 77, 3689-3692, 1996.

Sahimi, M., Robertson, M., and Sammis, C.: Fractal distribution of earthquakes hypocenters and its relation to fault patterns and percolation, Phys. Rev. Lett., 70, 2186-2189, 1993.

Saleur, H., Sammis, C., and Sornette, D.: Discrete scale invariance, complex fractal dimensions, and log-periodic fluctuations in seismicity, J. Geophys. Res., 101, 17 661-17 667, 1996 a.

Saleur, H., Sammis, C., and Sornette, D.: Renormalization group theory of earthquakes, Nonlin. Proces. Geophys., 3, 102-109, 1996 b.

Salminen, L., Tolvanen, A., and Alava, M.: Acoustic emission from paper fracture, Phys. Rev. Lett., 89, 185 503-1 1885 503-4, 2002.

Sammis, C. and Smith, S.: Seismic cycles and the evolution of stress correlation in cellular automaton models of finite fault networks, Pure Appl. Geophys., 155, 307-334, 1999.

Sammis, C. and Sornette, D.: Positive feedback, memory, and the predictability of earthquakes, PNAS, 99, 2501-2508, 2002.

Sammis, C., Sornette, D., and Saleur, H.: Complexity and earthquake forecasting, in Reduction and Predictability of Natural Disasters, SFI studies in the Sciences of complexity, edited by Rundle, J., Klein, W. and Turcotte, D., vol. XXV, 143-156, Addison-Wesley, Reading, Mass., 1996.

Sanchez, R., Newman, D., and Carreras, B.: Waiting-time statistics of self-organized-criticality systems, Phys. Rev. Lett., 88, 068 302/1-068 302/4, 2002.

Scholz, C.: The frequency-magnitude relation of microfracturing in rock and its relation to earthquakes, Bull. Seism. Soc. Am., 58, 399-415, 1968.

Schuster, H.: Deterministic Chaos, VCH, Weinheim, 1998.

Sobolev, G., Chelidze, T., Zavyalov, A., and Slavina, L.: Maps of expected earthquakes based on a combination of parameters, Tectonophysics, 193, 255-265, 1991.

Sornette, A. and Sornette, D.: Self-organized criticality and earthquakes, Europhys. Lett., 9, 197-202, 1989.

Sornette, D.: Self-organized criticality in plate tectonics, in: Spontaneous Formation of Space-Time Sructures and Criticality, edited by Riste, T. and Sherrington, D., 57-106, Kluwer Academic Publishers, 1991.

Sornette, D.: Critical Phenomena in Natural Sciences, Springer, 2000.

Sornette, D. and Helmstetter, A.: Occurrence of finite-time singularities in epidemic models of rupture, earthquakes and starquakes, Phys. Rev. Lett., 89(15), 158 501-1 - 158 501-4, 2002.

Sornette, D. and Le Floc'h, C.: Predictability of catastrophic events; a new approach for structural health monitoring, Structural Health Monitoring Review, in press, (http://arXiv.org/abs/condmat/0210418), 2003.
Sornette, D. and Sammis, C.: Complex critical exponents from renormalization group theory of earthquakes: Implications for earthquake predictions, J. Phys. I., 5, 607-619, 1995.

Sornette, D., Miltenberger, P., and Vanneste, C.: Statistical physics of fault patterns self-organized by repeated earthquakes, Pure and Applied Geophysics, 142, 491-527, 1994.

Stanley, H.: Scaling, universality, and renormalization: Three pillars of modern critical phenomena, Reviews of Modern Physics, 71, S358-S366, 1999.

Torrence, C. and Compo, P.: A practical guide to wavelet analysis, Bull. Amer. Meteor. Soc., 79, 61-78, 1998.

Tramutoli, V., Di Bello, G., Pergola, N., and Piscitelli, S.: Robust satellite techniques for remote sensing of seismically active areas, Annali di Geofisica, 44, 295-312, 2001.

Turcotte, D.: Fractal in geology and geophysics, in: Fractal in Geophysics, edited by Scholz, C. and Mandelbrot, B., 171, Birkhäuser, 1989.

Turcotte, D.: Fractals and chaos in geology and geophysics, Cambridge University Press, 1992.

Turcotte, D.: Self-organized criticality: does it have anything to do with criticality and is it useful?, Nonlin. Proc. Geophys., 8, 193196, 2001.

Tzanis, A. and Makropoulos, K.: Did the 7/9/ 1999 M5.9 Athens earthquake come with a warning?, Natural Hazard, 27, 85-103, 2002.

Varnes, D. and Bufe, C.: The cyclic and fractal seismic series preceding an $m_{b}=4.8$ earthquake on 1980 February 14 near the Virgin Islands, Geophys. J. Int., 124, 149-158, 1996.

Velde, B., Moore, D., Badri, A., and Ledesert, B.: Fractal and length analysis of fractures during brittle to ductile changes, J. Geophys. Res., 98, 11 935-11 940, 1993.

Vespignani, A., Petri, A., Alippi, A., Paparo, G., and Costantini, M.: Long range correlation properties of aftershock relaxation signals, Fractals, 3, 839, 1995.

Warwick, J., Stoker, C., and Meyer, T.: Radio emission associated with rock fracture: possible application to the great Chilean earthquake of May 22, 1960, J. Geophys. Res., 87, 2851-2859, 1982.

Weiss, J.: Fracture and fragmentation of ice: A fractal analysis of scale invariance, Engineering Fracture Mechanics, 68, 19752012, 2001.

Yamada, I., Masuda, K., and Mizutani, H.: Electromagnetic and acoustic emission associated with rock fracture, Physics of the Earth and Planetary Interiors, 57, 157-168, 1989.

Zoller, G. and Hainzl, S.: A systematic spatiotemporal test of the critical point hypothesis for large earthquakes, Geophys. Res. Lett., 29, 53/1-53/4, 2002.

Zoller, G., Hainzl, S., and Kurths, J.: Observations of growing correlation length as an indicator for critical point behavior prior to large earthquakes, J. Geophys. Res., 106, 2167-2175, 2001. 